\title{
Reliable Data Transportation in Wireless Sensor Networks
}

\author{
Mamta Katiyar \\ ECE Department \\ M. M. Engineering College, \\ M. M. University \\ Mullana, Ambala, Haryana, \\ India-133207
}

\author{
H.P.Sinha, PhD. \\ ECE Department \\ M. M. Engineering College, \\ M. M. University \\ Mullana, Ambala, Haryana, \\ India-133207
}

\author{
Dushyant Gupta, PhD. \\ Electronics Department, \\ University College, \\ KUK \\ Kurukshetra, Haryana, \\ India-136119
}

\begin{abstract}
A wireless sensor network (WSN) consists of sensor nodes and base stations which are connected via wireless medium. A key functionality of WSNs consists in collecting information from sensor nodes \& transporting the information of interest to the base stations required by the applications. Wireless connectivity, size and low cost of sensors in WSNs are its advantages which enable it to be deployed in hostile or inaccessible environments at a very low cost. However, WSNs suffer from high data loss due to error prone wireless transmission medium, transmission problems in hostile environments and node failures due to limited energy of sensor nodes. Hence reliable data transportation i.e. ensuring data delivery with minimum loss becomes the key issue in WSNs. The amount of loss tolerated is application dependent. This paper presents an artificial neural network based model for reliable data transportation in Wireless Sensor Networks (WSNs). This work increases the reliability of information transportation despite of corrupted signal sensed at the sensors and thus is an attempt to design a fault tolerant WSN.
\end{abstract}

Keywords: WSN, ANN, IS, PA.

\section{Introduction}

A wireless sensor network consists of very small and unobtrusive sensor nodes which configure themselves to aid the formation of the wireless network, and is inexpensive. Hence, wireless sensor networks can be deployed in areas which are difficult to access e.g. in enemy territory for surveillance purposes, animal/plant habitat monitoring in dense forests, within human body parts for monitoring diseases like cancer, etc. This has lead to increased application and research in the area of wireless sensor networks.

Network reliability and scalability have a very close relation and often act against each other. Therefore it is challenging to build a reliable network as the number of nodes increases [1]. This is because of network overhead that comes with increased size of network. Moreover, with the increased network, there is more possibility that communication links get broken.

Battery with limited Energy is also one of the key challenges to be considered for reliable data transportation in WSNs. By reducing the duty cycle of a node, we can take the advantage of power saving [2] but will have to pay for reliability due to reduced probability for communication with its neighbors.

Guaranteed action is required in many applications of WSNs like medical emergency alarm, fire detection, intrusion detection [3], the distribution of new code or queries from the sink node to sensors [4], tracking application between nodes close to the target trajectory [5], [6]. In these situations packets has to be delivered in a reliable manner through the sensor network. Thus, data reliability becomes very important for the proper functioning of the network. In this work we have proposed a neural network based model because of the following reasons: In a WSN platform, neural networks can help in lowering the communication cost and energy savings [7]. The other reason is the analogy between WSNs and ANNs. Authors in [8] recommended that Neural Networks architecture is similar to WSNs.

\section{PROPOSED SYSTEM MODEL}

\section{A. Data Forwarding Process}

Proposed model is an attempt to improve the fault tolerance and reliability of data transportation in WSN. This model addresses the two cases of unsuccessful data delivery at sink. i) Corrupted data delivery at the sink due to noisy channel, ii) Load balancing at the sink as packet may drop at the sink when it is receiving data from multiple sources at the same time.

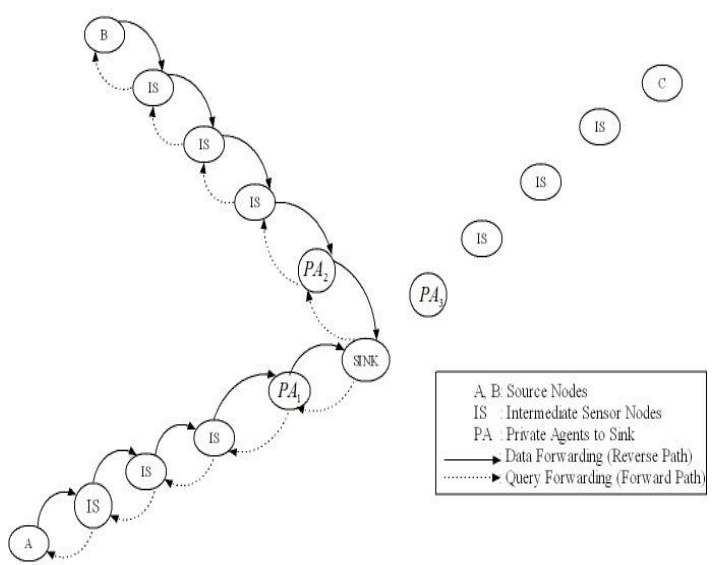

Fig 1: Query and data transportation between multiple sources and a sink

Fig 1 shows the communication process between source and sink. Sink S starts flooding its query with its private agents towards the various sources through the intermediate sensor nodes (IS) and continued until query reaches to the source. In the reverse path, data generated from source is first passed to the neural network, embedded in each sensor node, for encoding the related association to reduce the size of data/packet before forwarding to the next IS. Section 2.1 presents a neural network based reliable and fault tolerant 
mechanism for successful data delivery at the sink despite of corrupted data received during the reverse path. Private agent based load balancing scheme has been presented in Section 2.2 .

\subsection{System Model to Handle Corrupted Data}

The proposed model is most suitable for the applications where the events occurring in the network are from a predefined set of events. Consider that the sensor node is assumed to sense an event from a predefined set. Larger the size of a packet, more are the chances for faulty delivery of packet to the next probable forwarder/destination. In this model, all the sensors are assumed to be biologically inspired where a neural network is embedded in each sensor node. Fig 2 presents the structure and functionality of neural network embedded in each sensor nodes.

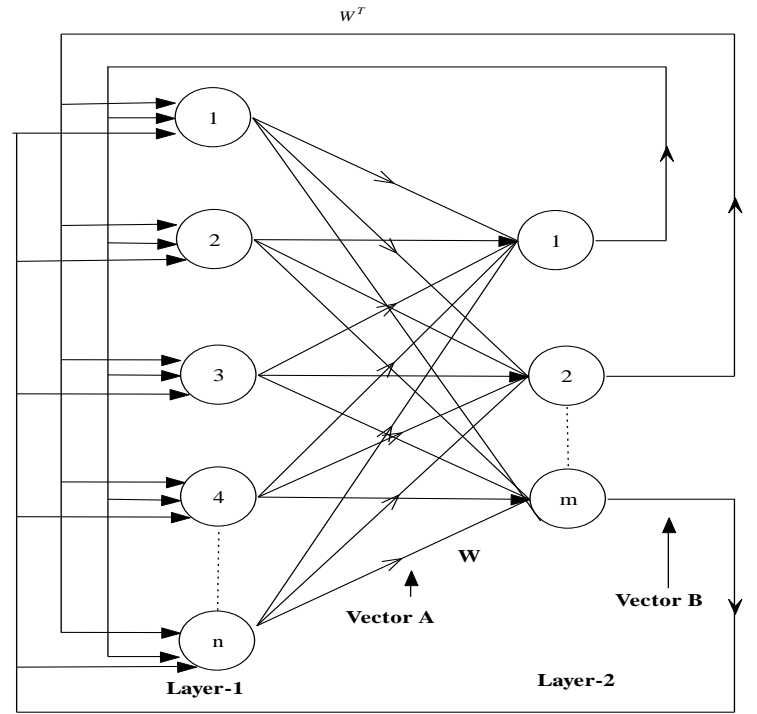

Fig 2: Architecture of Neural Network Embedded in sensor node

Data generated at the source is first given to the neural network to reduce the size of packets by encoding the associations before forwarding it to the next forwarder (IS) for onwards transmission towards the sink.

Proposed neural network is embedded in every sensor node of WSN as shown in Fig. 2. This associative network is a hetroassociative recurrent neural network consisting of two layers. Layer-1 has $n$ units and layer-2 has m units. Synaptic strength of various connections from the layer- 1 to layer- 2 is represented by the weight matrix $\mathrm{W}_{\mathrm{nXm}}$ and the weight matrix from layer-2 to layer-1 is $W_{n x m}^{T}$. This is a fully connected network, wherein the inputs are different from the outputs. In the forward pass, weight matrix $\mathrm{WnXm}$ is used to reduce the size of packet whereas $W_{n x m}^{T}$ is used in the reverse pass at private agent to retrieve the original packet sensed at the source. After training the network, if vector A is presented over the network then it recalls the associated vector B and similarly vector B recalls vector A if vector B is presented over the transpose weight matrix $W^{T}$.

\section{B. Training procedure}

Suppose we want to encode the associations between the hetro-associative patterns for the given problem space.

\begin{tabular}{|c|c|}
\hline $\begin{array}{c}\text { Vector A } \\
\text { (Actual packet of size n) }\end{array}$ & $\begin{array}{c}\text { Associated Vector } \\
B\end{array}$ \\
\hline Training pair 1:A & $B_{1}$ \\
\hline Training pair 2: $A_{2}$ & $B_{2}$ \\
\hline & $\vdots$ \\
\hline Training pair : $A_{k}$ & $B_{k}$ \\
\hline
\end{tabular}

Binary Version
Bipolar Version

\begin{tabular}{|c|c|}
\hline $\begin{array}{c}\text { Vector } \mathrm{A} \\
\text { (Actual packet of size } \mathrm{n})\end{array}$ & $\begin{array}{c}\text { Associated Vector } \\
\mathrm{B}\end{array}$ \\
\hline Training pair $1: A_{1}^{\prime}$ & $B_{1}^{\prime}$ \\
\hline Training pair 2: $A_{2}^{\prime}$ & $B_{2}^{\prime}$ \\
\hline Training pair $\mathrm{k}: A_{k}^{\prime}$ & $B_{k}^{\prime}$ \\
\hline
\end{tabular}

Each training pair is applied on the network and weight is adjusted in a manner so that if we apply vector A then the network should recall associated vector $\mathrm{B}$ and vice versa. After the training, weight matrix

$$
W=\sum_{i=1}^{k}\left(A_{i}^{\prime}\right)^{T} B_{i}^{\prime} .
$$

At all the source nodes, before transmitting a packet $\mathrm{Ai}$, it is presented over the network through weight matrix $\mathrm{W}$ to get a smaller associated vector $\mathrm{Bi}$. Associated vector $\mathrm{Bi}$ is transmitted and is more reliably delivered at the next forwarder for onward transmission. When the associated vector is received at the private agent, it is passed to the neural network in the reverse pass of the network through $\mathrm{W}^{\mathrm{T}}$ to ensure retrieval of the actual packet $A_{i}$. Received packet is recalled at PA for onward transmission to sink if packet is not corrupted beyond the fault tolerance of network otherwise a retransmission request is initiated by the PA. Activation function used in the proposed neural network is described as:

$$
\text { Output }=\mathrm{f}(\mathrm{NET})=\left\{\begin{array}{l}
1, \text { if NET }>0 \\
0, \text { if NET }<0 \\
\text { Previous output, if NET }=0
\end{array}\right.
$$

Where NET $= \begin{cases}\text { A. } W & \text { at Layer }-2 \\ \text { B. } W^{\mathrm{T}} & \text { at Layer }-1\end{cases}$

The memory capacity of this neural network is defined as: $\min (n, m)$; where $n$ is the number of units in layer-1 (size of vector $A$, i.e., the original packet) and $m$ is the number of units in layer-2 (size of vector B, i.e., the associated vector to be transmitted). This network is capable for reliable and fault tolerant transmission within its maximum memory capacity even if the number and size of data packets is very large.

\subsection{Agent Based Load Balancing at Sink}

In the proposed model, each PA embedded with the neural network works in the reverse pass to retrieve the original packet transmitted from the source. PAs are used to reduce the load at the sink when a sink is receiving data from multiple sources at the same time. Consider the shared private agent cluster system shown in Fig. 3. The sensors ability to share the private agent cluster provides a convenient means of sharing information and provides flexibility in PA allocation for further processing of information before delivering it to sink. When more than one IS attempts to access the same PA, only one IS can be granted access while others must wait for their turn in a queue. The effect of such contention or interference is to increase the average PA access time. 


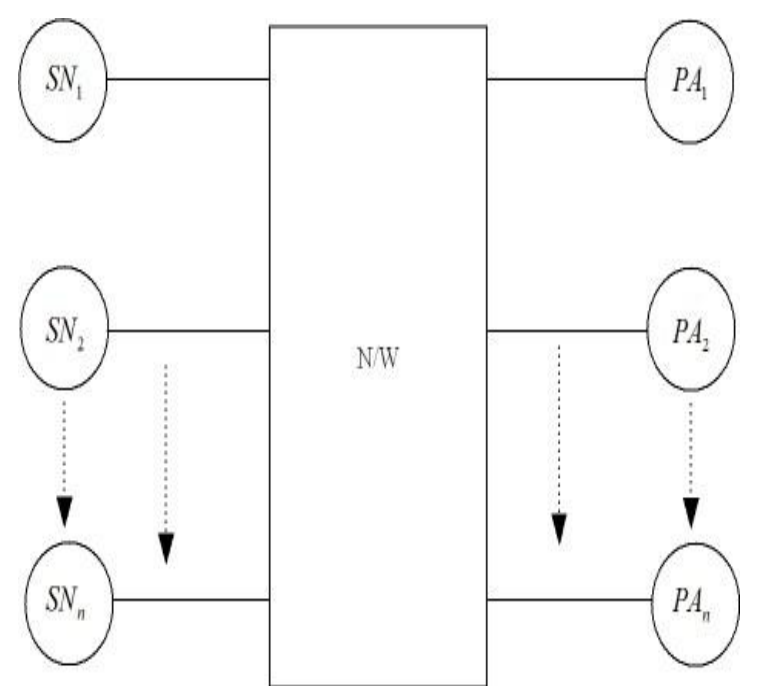

Fig 3: A Shared Private Agent System

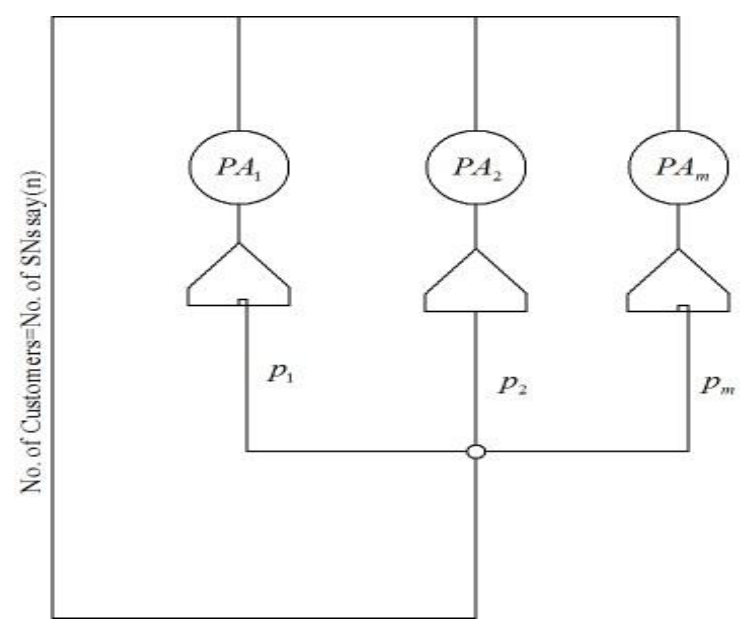

Fig 4: Queuing N/W for multisensory nodes

Assume that time to complete a PA access is a constant and that all PAs are synchronized. IS are assumed fast enough to generate a new request as soon as their current request is satisfied. An IS cannot generate a new request when it is waiting for the current request to be satisfied. The functionality of the system can be visualized as shown in Fig 4

In figure, PAs are the servers and sensor nodes constitute the job circulating in the queuing network. Assuming pi is the probability that a sensor node generated request is directed at private agent $\mathrm{i}, \mathrm{i}=1,2, \ldots, \mathrm{m}$, then $\sum_{i=1}^{m} p_{i}=1$.

\subsubsection{Analysis of system model with three IS and two PAs at Sink}

Considering a sink with two PAs and three ISs from where it has to collect the information of interest. The pair (si,s2) denotes the state of the system and the state space $\mathrm{I}=\{(3,0),(0,3),(1,2),(1,2)\}$. The functionality of the system can be described by the state transition diagram shown in Fig 5 .

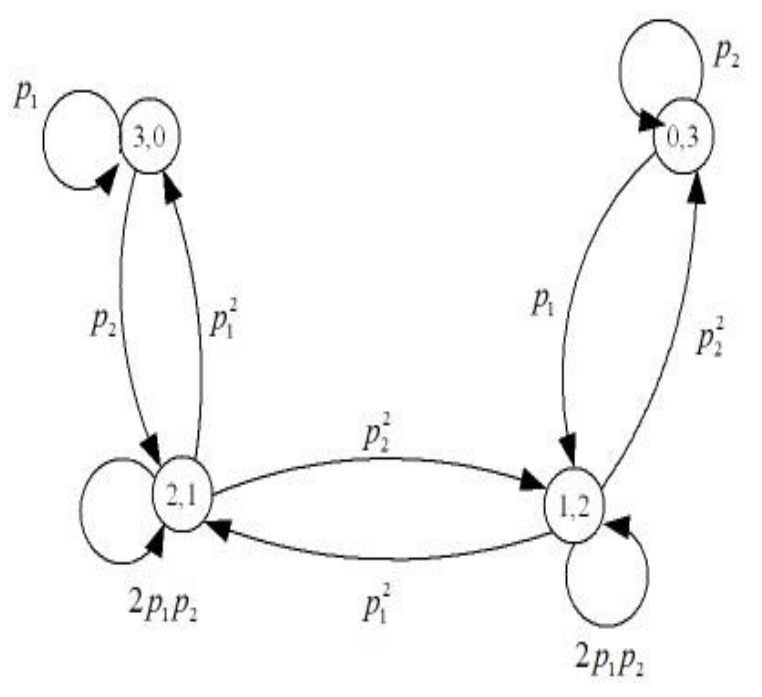

Fig 5: State Transition Diagram for a Sink with Three IS and Two PAs

State transition probability matrix is given by

\begin{tabular}{c|cccc}
\multicolumn{1}{c}{$v_{3,0}$} & $v_{2,1}$ & $v_{1,2}$ & $v_{0,3}$ \\
\cline { 2 - 5 }$v_{3,0}$ & $p_{1}$ & $p_{2}$ & 0 & 0 \\
$v_{2,1}$ & $p_{1}^{2}$ & $2 p_{1} p_{2}$ & $p_{2}^{2}$ & 0 \\
$v_{1,2}$ & 0 & $p_{1}^{2}$ & $2 p_{1} p_{2}$ & $p_{2}^{2}$ \\
$v_{0,3}$ & 0 & 0 & $p_{1}$ & $p_{2}$
\end{tabular}

To get the steady state probabilities

$$
\begin{aligned}
& p_{1} v_{3,0}+p_{1}^{2} v_{2,1}=v_{3,0} \\
& p_{2} v_{3,0}+2 p_{1} p_{2} v_{2,1}+p_{1}^{2} v_{1,2}=v_{2,1} \ldots \\
& p_{2}^{2} v_{2,1}+2 p_{1} p_{2} v_{1,2}+p_{1} v_{0,3}=v_{1,2} \ldots \\
& p_{2}^{2} v_{1,2}+p_{2} v_{0,3}=v_{0,3} \\
& v_{3,0}+v_{2,1}+v_{1,2}+v_{0,3}=1 \\
& v_{0}=\frac{\left(p_{2}^{5}\right)}{\alpha} \\
& v_{1}=\frac{p_{1} p_{2}^{3}}{\alpha} \\
& v_{2}=\frac{p_{1}^{3} p_{2}}{\alpha} \\
& v_{3}=\frac{p_{1}^{5}}{\alpha}
\end{aligned}
$$


Where $\alpha=\left(p_{1}^{5}+p_{2}^{5}+p_{1}^{3} p_{2}+p_{1} p_{2}^{3}\right)$

Let $\mathrm{X}$ be a random variable representing the number of requests completed per cycle in the steady state. Our aim is to compute the coverage no of requests completed per cycle, $\mathrm{E}[\mathrm{X}]$, where $\mathrm{E}[\mathrm{X}]$ is expectations of random variable $\mathrm{X}$. the conditional expectations of $\mathrm{X}$ are given by

$\{\mathrm{E}[\mathrm{X}] \mid$ system is in state $(3,0)\}=1$

$\{\mathrm{E}[\mathrm{X}] \mid$ system is in state $(2,1)\}=2$

$\{\mathrm{E}[\mathrm{X}] \mid$ system is in state $(1,2)]=2$

$\{\mathrm{E}[\mathrm{X}] \mid$ system is in state $(0,3)]=1$

Therefore total expectations are

$$
E[X]=1 * v_{(3,0)}+2 * v_{(2,1)}+2 * v_{(1,2)}+1 * v_{(0,3)}
$$

$\mathrm{E}[\mathrm{X}]$ achieves its maximum value, approximately 1.67 when $\mathrm{p} 1=\mathrm{p} 2=1 / 2$. Though it is smaller than the maximum capacity of the system (2 requests per cycle) but is far better than the worst case of system performance i.e. one request per cycle.

Similarly if we consider a system model with two IS and two $\mathrm{PAs}$ at sink then $\mathrm{E}[\mathrm{X}]$ achieves its maximum value, 1.5, which is smaller than the capacity of the system i.e. 2 requests per cycle.

\section{CONCLUSIONS}

In this paper, we proposed a fault tolerant and reliable WSN model based on the novel concept of encoding associations among the hetro-associative vectors using artificial neural networks. Reliability and fault tolerance model for corrupted data has been illustrated in section 2.1. In this paper we have also proposed a model for reliable data transportation by load balancing at sink. Performance of this model has been analyzed in section 2.2.1 and it has been concluded that the reliability of the network can be increased by increasing the number of PAs at sink in case it is receiving data from multiple sources at the same time.

\section{REFERENCES}

[1] W. Cai, X. Jin, Y. Zhang, K. Chen, J. Tang, "Research on Reliability Model of Large-Scale Wireless Sensor Networks", in Proceeding of Wireless Communications, Networking and Mobile Computing (WiCOM 2006) pp. $1-4,2006$.

[2] C. F. Chiasserini, M. Garetto, "An analytical model for wireless sensor networks with sleeping nodes", IEEE Trans. Mobile Computing, vol. 5(12), pp. 1706-1718, 2006.

[3] Bernardo, R. Oliveria, R. Tiago, P. Pinto, "A Fire Monitoring Application for Scattered Wireless Sensor Network", in the proceeding of WinSys 2007, on July 28-31, 2007.

[4] C.-Y. Wan, A. T. Campbell, and L. Krishnamurthy, "Psfq: A reliable transport protocol for wireless sensor networks," in Proc. First ACM Intl. Workshop on Wireless Sensor Networks and Applications (WSNA'02), Atlanta, GA, 2002.

[5] R. R. Brooks, P. Ramanathan, and A. M. Sayeed, "Distributed Target Classification and Tracking in Sensor Networks,"Proceedings of the IEEE, vol. 91, no. 8, pp. 1163-1171, Aug. 2003.

[6] F. Zhao, J. Shin, and J. Reich, "Information-driven dynamic sensor collaboration," IEEE Signal Processing Magazine, pp. 61-72, Mar. 2002.

[7] A. Kulakov, D. Davcev, G. Trajkovski, "Application of wavelet neural-networks in wireless sensor networks", Sixth International Conference on Software Engineering, Artificial Intelligence, Networking and Parallel/Distributed Computing and First ACIS International Workshop on Self-Assembling Wireless Networks (SNPD/SAWN'05), pp.262-267, 2005.

[8] Oldewurtel, Frank, Mahonen, Petri, "Neural Wireless Sensor Networks", International Conference on systems \& Networks Communications, ICSNS'06, pp 28-28. 